\title{
Filigrane
}

Écoutes psychothérapiques

\section{Plaidoyer pour une psychanalyse empathique}

\section{Serge Tisseron}

Volume 23, numéro 1, printemps 2014

Qu'est la psychanalyse devenue?

URI : https://id.erudit.org/iderudit/1026077ar

DOI : https://doi.org/10.7202/1026077ar

Aller au sommaire du numéro

Éditeur(s)

Revue Santé mentale au Québec

ISSN

1192-1412 (imprimé)

1911-4656 (numérique)

Découvrir la revue

\section{Citer cet article}

Tisseron, S. (2014). Plaidoyer pour une psychanalyse empathique. Filigrane, 23(1), 51-64. https://doi.org/10.7202/1026077ar

\section{Résumé de l'article}

Après avoir évoqué l'évolution des pathologies, l'auteur rappelle que Sándor Ferenczi, dès 1928, a posé le concept d'empathie comme un outil majeur du psychanalyste. Prenant en compte les plus récents travaux à ce sujet, il montre ensuite que ce concept se laisse décomposer en trois dimensions. La première, l'empathie directe, est un mode de connaissance d'autrui qui permet de se mettre à la place de l'autre tout en restant conscient de la différence entre lui et soi. La seconde dimension est l'empathie réciproque, qui ajoute à la possibilité de se mettre à la place de l'autre le principe d'une réciprocité possible. Enfin, la troisième dimension correspond à ce qui est couramment appelé intersubjectivité, et qui consiste à reconnaître à l'autre la possibilité de nous éclairer sur des aspects de soi que l'on ignore. C'est cette dernière dimension que l'analyste doit avoir à coeur de développer. Sur ce chemin, l'auteur propose de penser la psychanalyse sur le modèle d'une relation de compagnonnage : mutuelle, réciproque et non symétrique.
Ce document est protégé par la loi sur le droit d'auteur. L'utilisation des services d'Érudit (y compris la reproduction) est assujettie à sa politique d'utilisation que vous pouvez consulter en ligne.

https://apropos.erudit.org/fr/usagers/politique-dutilisation/ 


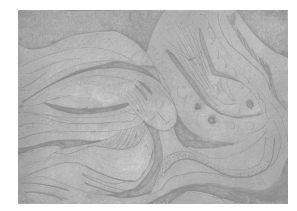

\title{
Plaidoyer pour une psychanalyse empathique
}

\author{
SergeTisseron
}

Après avoir évoqué l'évolution des pathologies, l'auteur rappelle que Sándor Ferenczi, dès 1928, a posé le concept d'empathie comme un outil majeur du psychanalyste. Prenant en compte les plus récents travaux à ce sujet, il montre ensuite que ce concept se laisse décomposer en trois dimensions. La première, l'empathie directe, est un mode de connaissance d'autrui qui permet de se mettre à la place de l'autre tout en restant conscient de la différence entre lui et soi. La seconde dimension est l'empathie réciproque, qui ajoute à la possibilité de se mettre à la place de l'autre le principe d'une réciprocité possible. Enfin, la troisième dimension correspond à ce qui est couramment appelé intersubjectivité, et qui consiste à reconnaître à l'autre la possibilité de nous éclairer sur des aspects de soi que l'on ignore. C'est cette dernière dimension que l'analyste doit avoir à cœur de développer. Sur ce chemin, l'auteur propose de penser la psychanalyse sur le modèle d'une relation de compagnonnage: mutuelle, réciproque et non symétrique.

\begin{abstract}
$\Lambda$ près avoir été hégémonique dans toutes les institutions de soins, la psychanalyse est aujourd'hui en crise. Il ne s'agit pas seulement d'une crise de légitimité, nourrie par des cliniciens et des associations familiales qui l'accusent, avec plus ou moins de bonne foi, d'être restée fermée aux récentes découvertes, notamment sur l'autisme. Il s'agit d'abord d'une crise théoricoclinique, liée à l'évolution des pathologies et à la difficulté d'un nombre croissant de psychanalystes, notamment parmi les plus jeunes, à se reconnaître dans le modèle de relation thérapeutique que leur ont transmis leurs maîtres. Un modèle fait souvent de distance, voire de froideur, dans lequel la fameuse bienveillance que Freud plaçait au cœur de la neutralité analytique semble avoir disparu. Cette façon de pratiquer la psychanalyse s'est d'autant plus facilement imposée qu'elle semblait correspondre à une époque, les années 1960 à 1990, pendant lesquelles la prospérité économique et les certitudes idéologiques conduisaient beaucoup de patients à y rechercher un espace
\end{abstract}


propre à les déstabiliser afin de leur permettre de se rendre disponible à de nouveaux désirs que leur éducation avait brimés. Mais aujourd'hui, la situation a bien changé. D'une part, de nouvelles formes de souffrances apparaissent, sur fond de crise économique et de perte des repères familiaux. Elles sont en lien avec des situations professionnelles de plus en plus éprouvantes et une instabilité affective qui est devenue la règle. Lorsque ces situations entrent en résonance avec des carences éducatives précoces ou des traumatismes de toutes natures, elles affectent la confiance en soi et dans le monde avec des risques de repliement ou de conduites extrêmes. Mais il n'y a pas que les pathologies qui aient changé. Les attentes aussi ont considérablement évolué. L'utilisation intensive de l'internet suscite en effet dans toutes les générations un état d'esprit qui s'accommode mal d'une relation d'autorité et qui valorise l'échange et la confrontation.

Face à ces nouvelles formes de souffrances et de modalités relationnelles, certains psychanalystes cherchent à justifier des formes de pratiques qui les éloignent de plus en plus de la réalité des pathologies et de leurs aspirations. D'autres, notamment parmi les plus jeunes, se demandent comment travailler autrement. C'est pour leur répondre qu'il est urgent de rappeler que la psychanalyse porte en elle un fort pouvoir d'adaptation et d'évolution, à condition de la ramener à l'essentiel du message freudien : constituer un lieu de subjectivation et d'appropriation, par chaque patient, de son histoire personnelle. Autrement dit, une fois le cadre fixé (dont la fréquence des séances et leur paiement sont les deux éléments majeurs), pourquoi ne pas établir avec un patient une relation empathique qui lui permette de reprendre confiance en lui et dans le monde, puisque c'est souvent cette souffrance-là qui est mise en avant? Peut-être certains penseront-ils qu'une telle relation est incompatible avec la situation analytique qui pose une dissymétrie fondamentale entre l'analyste et son patient. Pourtant, l'asymétrie d'une relation n'est pas un obstacle à la réciprocité. C'est ce que montre admirablement le film Intouchables ${ }^{1}$, qui a bouleversé tous ceux qui l'ont vu. Rappelons qu'il s'agit de l'histoire d'un homme tétraplégique, Philippe, qui cherche une sorte d'ange gardien qui le nourrisse, le masse, le promène et veille sur lui jour et nuit. L'un des candidats au poste s'appelle Driss : c'est un jeune Noir rebelle. Philippe le choisit. Le spectateur est d'abord choqué. Driss n'a en effet aucune pitié pour Philippe. Mais c'est justement pour cela que Philippe l'a choisi! Et c'est même l'un des messages les plus forts de ce film. Rien n'est plus insupportable à la personne handicapée que la pitié. Si Driss n’a pas de pitié, il a autre chose: de l'empathie. Bien sûr, à vouloir être trop consensuel et 
commercial, ce film ignore tous les aspects socioéconomiques de la question du handicap. Mais sa leçon reste essentielle: une relation authentique repose sur une empathie mutuelle et réciproque, et cette relation n'a pas besoin d'être symétrique. De même, une relation thérapeutique fonctionne mieux quand le thérapeute est capable d'empathie.

\section{Ferenczi, un précurseur}

En psychanalyse, le concept d'empathie a un précurseur: Sándor Ferenczi. Certains l'ont accusé d'être allé un peu trop loin dans les rapprochements dans le réel, puisqu'il embrassait parfois ses patient(e)s, c'est-à-dire qu'il leur faisait une bise. Cela lui a valu une mise au point par Freud sur le danger de telles manœuvres. Hélas, justifiée ou non, cette mise au point a enrayé beaucoup d'aspects stimulants de l'œuvre de Ferenczi, notamment l'importance du «tact» en psychanalyse ${ }^{2}$. Pourtant, Freud avait lui-même écrit, dans une lettre à Sándor Ferenczi du 4 janvier 1928, à propos de son article sur «le tact»: «[...] les conseils sur la technique que j'ai écrits il y a longtemps ont essentiellement un caractère négatif. J'ai considéré qu'il fallait avant tout souligner ce que l'on ne devait pas faire et mettre en évidence les tentations capables de contrarier l'analyse. J'ai négligé de parler de toutes les choses positives qu'il faudrait faire et en ai laissé le soin au "tact" dont aujourd'hui vous entreprenez l'étude. Il en résulta que les analystes dociles ne saisirent pas l'élasticité des règles que j'avais formulées et qu'ils y obéissent comme si elles étaient taboues». Nous pourrions ajouter que les psychanalystes avaient très peur de sortir d'une orthodoxie rassurante et confortable... Mais cela a-t-il vraiment changé aujourd'hui ? Pour désigner le tact qu'il prônait, Ferenczi utilisait le mot Einfühlung, qui est traduit aujourd'hui par «empathie». Revenons alors sur ses conseils. Ferenczi insistait sur le renoncement à une attitude distante et inaffective qui se montre nécessaire pour créer et entretenir un lien avec ses patients. Il parlait de l'importance du tact (empathie) dans la relation thérapeutique qui nous contraint à ne jamais imposer à nos patients nos propres idées, voire nos propres a priori, et, pour cela, de créer les conditions d'une réciprocité. Aujourd'hui, il est possible de donner au mot empathie une définition plus complète qu'à l'époque de Ferenczi.

Pour comprendre ce qu'est l'empathie, commençons d'abord par parler de ce qu'elle n'est pas, à savoir ni la sympathie, ni la compassion, ni l'identification. Dans la sympathie, on partage non seulement les mêmes émotions, mais aussi les valeurs, les objectifs et les idéaux de l'autre. C'est ce que signifie 
le mot «sympathisant». La compassion, elle, met l'accent sur la souffrance. Elle est inséparable de l'idée d'une victime et du fait de prendre sa défense contre une force hostile, voire une agression humaine. Son principal danger est qu'elle fait peu de place à la réciprocité, et s'accompagne même parfois d'un sentiment de supériorité.

Alors, qu'est-ce que l'empathie? Dans sa définition la plus commune, elle est le fait de s'identifier à quelqu'un du point de vue de ce qu'il ressent. Elle ne suffit évidemment pas à établir une relation complète avec quelqu'un. Il y faut d'autres registres: la compréhension des états mentaux (et pas seulement émotionnels) de l'autre, la reconnaissance de ses droits, et même la capacité d'accepter que l'autre puisse m'informer de choses que j'ignore sur moi-même. Nous pourrions considérer ces diverses composantes de la relation comme étant séparées. Pourtant, chacun sent bien qu'elles font un tout. C'est pourquoi j'ai proposé, en 2010, d'élargir le sens du mot «empathie» pour y faire entrer ces diverses composantes, et de parler «des empathies» plutôt que de «l'empathie». La capacité de s'identifier à quelqu'un du point de vue de ce qu'il ressent n'est alors que le premier degré d'un processus qui en comporte trois. Et l'ensemble peut être représenté sous la forme d'une pyramide constituée de trois étages superposés: en bas se trouve l'empathie directe qui apparaît en premier dans le développement de l'enfant, avec ses deux composantes émotionnelle et cognitive; au milieu se trouve l'empathie réciproque; et en haut l'empathie à la fois réciproque et mutuelle, qui correspond à ce qu'on appelle aussi l'intersubjectivité.

D'autres choix sémantiques sont possibles. Le plus courant consiste à penser l'ensemble de ces étages sous le mot «compassion », pris non plus au sens qu'il a dans la tradition chrétienne, mais au sens qu'il a dans la tradition bouddhiste : l'être humain y est considéré comme en souffrance permanente par rapport à son désir d'un bonheur toujours impossible. Le mot compassion ne désigne plus alors la miséricorde et la pitié, mais l'humanité et la sensibilité, et il recouvre la plupart des phénomènes que nous regroupons ici sous le mot d'empathie. À la suite des travaux de Francisco Varela, cette perspective a été adoptée par des chercheurs qui désirent légitimer par les neurosciences les bienfaits des exercices spirituels du bouddhisme tibétain. Pour eux, le mot d'empathie désigne uniquement la composante émotionnelle de l'empathie directe, et toutes les autres composantes de ce que nous désignons comme diverses formes d'empathie sont regroupées sous le mot compassion (c'est-à-dire la composante cognitive de l'empathie directe, l'empathie réciproque et l'empathie à la fois réciproque et mutuelle). 
Il existe toutefois entre ces deux approches un point commun essentiel: le premier étage de la compassion dans la tradition bouddhiste nécessite qu'une claire distinction entre soi et l'autre ait été établie, exactement comme le premier étage de l'empathie dans notre approche (celui que nous appelons empathie directe). Si ce n'est pas le cas, on parle de contagion émotionnelle ou de sympathie, et ce processus est mis en relation avec le rôle des «neurones miroirs » qui, comme leur nom l'indique, font adopter une attitude en miroir de celle de notre interlocuteur: quand il pleure, nous pleurons, et quand il rit, nous rions. Si certains lecteurs préfèrent penser en termes de compassion plutôt que d'empathie réciproque et d'intersubjectivité, rien ne sera donc changé pour eux dans la description des phénomènes que nous allons évoquer... sauf quelques mots.

Voyons maintenant notre schéma des trois étages de l'empathie. Après lui avoir d'abord donné la forme d'une pyramide (2010), nous avons finalement opté pour celle d'un bateau afin de souligner que c'est un processus toujours dynamique.

\section{L'empathie directe (ou identification empathique)}

En bas se trouve la quille, que tous les bateaux partagent: c'est l'empathie directe qu'on trouve chez tous les êtres humains, et aussi chez certains animaux, notamment les singes supérieurs. Elle se définit comme la capacité de changer de point de vue sans s'y perdre. Elle a deux composantes : l'une, l'empathie émotionnelle, consiste à s'imaginer ce qu'on pourrait éprouver à la place de l'autre et à le ressentir partiellement; l'autre, l'empathie cognitive, consiste à s'imaginer ce qu'on penserait si on était à la place de l'autre. La première amène un singe bonobo à cesser de se nourrir en appuyant sur un levier s'il découvre qu'un congénère placé dans une autre cage à côté de la sienne reçoit une décharge électrique à chaque fois qu'il appuie sur le levier. La seconde lui permet de faire semblant d'aller chercher des bananes là où il n'y en a pas pour entraîner ses congénères sur une fausse piste afin de garder sa découverte pour lui tout seul.

Les bases de cette capacité sont neurophysiologiques et elles sont toujours assurées chez les humains, sauf en cas de trouble mental autistique. L’empathie émotionnelle, parfois appelée intelligence émotionnelle, apparaît au cours de la première année de la vie, plus ou moins précocement selon les auteurs, lorsque le bébé commence à faire la distinction entre l'autre et lui. Elle permet d'identifier les émotions ressenties par un interlocuteur et les siennes propres, et d'y réagir de façon appropriée. Quant à l'empathie 
cognitive (si nous faisons le choix de l'appeler ainsi), elle apparaît aux alentours de quatre ans et demi : c'est ce qu'on appelle couramment la théorie de l'esprit.

En principe, les deux sont liées et constituent ensemble la capacité d'identification. Lorsque nous comprenons le point de vue de l'autre en nous identifiant à lui, nous ressentons en même temps ce qu'il ressent. Ce lien serait toutefois inexistant (ou rompu?) chez les personnes qu'on appelle «psychopathes »: elles auraient la capacité d'utiliser leur compréhension d'autrui pour le manipuler ou l'exploiter sans rien ressentir de ses souffrances.

Mais de façon générale, la capacité d'identification, dans sa double composante émotionnelle et cognitive, ne nécessite pas de reconnaître à l'autre la qualité d'être humain. La preuve en est qu'on peut s'identifier à un héros de dessin animé ou de roman.

Enfin, l'empathie ainsi définie peut tout autant être mise au service de la réciprocité que de l'emprise. Dans le premier cas, elle suscite l'entraide et la solidarité tandis que dans le second, elle entretient des formes parfois très subtiles de manipulation des esprits et des consciences. En outre, à partir de l'âge de sept ans, l'enfant a la possibilité d'inhiber son empathie pour autrui. Le problème principal de l'empathie n'est pas comment on la développe, mais les raisons sociales qui amènent chacun à pouvoir l'inhiber.

\section{L'empathie réciproque}

Pour prendre en compte la complexité des relations humaines, il nous faut faire intervenir une autre dimension. Nous choisissons de l'appeler «empathie réciproque» car elle nous paraît complémentaire à l'identification empathique, la réciprocité en plus. Cette construction n'est pas nécessaire au navire empathie, qui peut flotter sans elle, mais elle montre que le navire est habité par des êtres humains. Elle relève donc d'un choix moral. Cette empathie-là ajoute à la possibilité d'avoir une représentation du monde intérieur de l'autre, le désir d'une reconnaissance mutuelle, et l'acceptation du fait que passer par l'autre est la meilleure - et la seule? - façon de se connaître soi-même. Non seulement je m'identifie à l'autre, mais je lui accorde le droit de s'identifier à moi, autrement dit de se mettre à ma place et, ainsi, d'avoir accès à ma réalité psychique, de comprendre ce que je comprends et de ressentir ce que je ressens. Nous acceptons de percevoir les autres hommes comme pourvus de sensibilité au même titre que nous et non pas comme de simples choses. Cette reconnaissance permet, comme l'a montré Emmanuel Levinas (2004), de concilier asymétrie et réciprocité. Elle renvoie 
à l'expérience du miroir. Elle implique un contact direct ainsi que tous les gestes expressifs, tels que le sourire, le regard croisé et les expressions faciales, par lesquels j'atteste accepter de faire de l'autre un partenaire d'interactions émotionnelles et motrices. Inversement, l'absence de cette médiation expressive revient à nier l'existence de l'autre.

Cette reconnaissance réciproque a trois composantes complémentaires: reconnaître à l'autre la possibilité de s'estimer lui-même comme je le fais pour moi (c'est la composante du narcissisme) ; lui reconnaître la possibilité d'aimer et d'être aimé (c'est la composante des relations d'objet); lui reconnaître la qualité de sujet de droit (c'est la composante de la relation au groupe $)^{3}$.

\section{L'empathie réciproque et mutuelle (ou intersubjectivité)}

Enfin, au-dessus se trouve la cheminée qui, pour les enfants, indique que le navire a une source d'énergie propre et peut avancer. Dans le navire empathie, ce troisième étage indique que l'empathie est une force qui pousse au lien, c'est-à-dire à la relation intersubjective. Ce désir de validation par le regard d'autrui trouve son origine au début de la vie lorsque le bébé cherche une approbation de lui-même dans les yeux de sa mère. Il nous accompagne ensuite tout au long de la vie, et il trouve aujourd'hui dans les nouvelles technologies un support privilégié d'expression et de mise en scène (Tisseron, 2008). Dans tous les cas, il suppose que je reconnaisse à autrui le pouvoir de m'informer utilement sur des aspects de moi-même qui me sont encore inconnus. Il ne s'agit plus seulement de s'identifier à l'autre, ni même de reconnaître à l'autre la capacité de s'identifier à soi en acceptant de lui ouvrir ses territoires intérieurs, mais de se découvrir à travers lui différent de ce que l'on croyait être, et de se laisser transformer par cette découverte. 


\section{Les trois étages de l'empathie (Tisseron, 2010)}

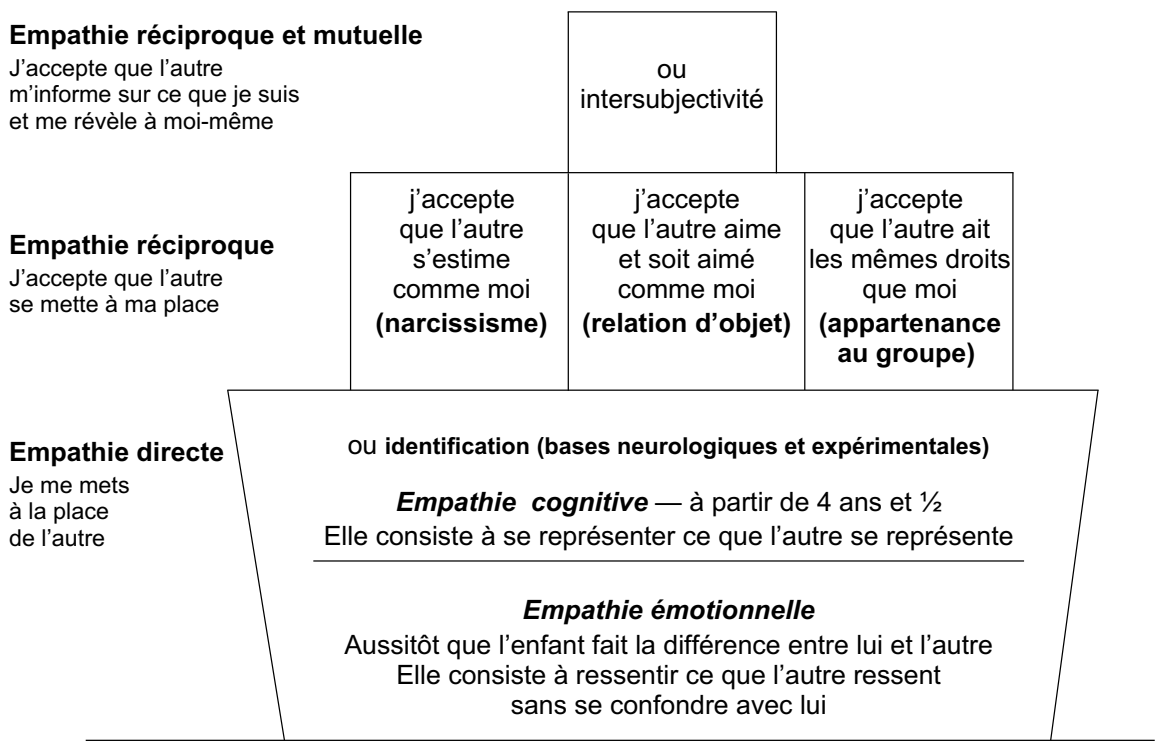

Cette empathie n'est plus seulement réciproque, elle est aussi mutuelle. Chacun apporte ce qu'il possède et profite de ce que les autres apportent à la mesure de ses besoins; elle n'est donc pas forcément symétrique. Le regard n'y est plus nécessaire, mais il l'a toujours été précédemment afin que l'empathie comme reconnaissance ait été posée. Alors, les parcours de vie différents de deux interlocuteurs sont une source d'enrichissement pour chacun. Puissent les thérapeutes ne jamais l'oublier!

\section{L'empathie pour reprendre confiance en soi et dans le monde}

L'enfant a besoin d'empathie dès sa naissance. Il a besoin que les adultes qui s'occupent de lui sachent identifier ses attentes, donner du sens à ce qu'il manifeste, et valoriser ses manifestations émotionnelles et verbales adaptées aux situations. C'est la condition pour qu'il croie en la valeur des représentations personnelles qu'il se construit. Et cela ne s'arrête pas avec l'enfance. Toute la vie, nous cherchons un interlocuteur qui nous écoute et nous comprenne. Et la plus sûre façon de penser que nous l'avons trouvé est le fait qu'il semble partager nos émotions d'une façon visible sur son visage. La preuve en est l'importance que prennent pour chacun d'entre nous les hochements de tête de nos interlocuteurs lorsque nous leur parlons de quelque chose 
d'important. C'est que nous avons besoin, pour trouver le courage d'explorer des aspects douloureux de nous-mêmes, de nous sentir accompagnés et soutenus. Mais si l'enfance est si importante, c'est parce qu'elle est le moment où la conviction de pouvoir trouver un tel interlocuteur se met en place.

En pratique, cela passe par le fait que l'entourage de l'enfant est capable de se réjouir avec lui de ce qui lui fait plaisir, et de s'attrister avec lui de ce qui le peine. C'est ce que font spontanément la plupart des parents. Ils savent percevoir la signification des manifestations émotionnelles de leur jeune enfant et y réagir de façon adaptée. Quand il rit, ils l'accompagnent de leur sourire, et quand il est inquiet, ils savent le prendre dans leurs bras et le serrer contre eux. Ces interactions rassurantes engagent l'enfant vers une confiance dans le monde et dans lui-même équilibrée et saine. Il apprend à faire confiance à sa compréhension du monde, et à croire que les autres peuvent l'aider à se comprendre lui-même. Il intériorise la possibilité de se rassurer et, plus tard, de rassurer les autres. C'est un peu comme s'il se disait: «Finalement, je n'ai que des avantages à montrer mes émotions à mes proches: ils me répondent avec les leurs, et nous passons d'excellents moments ensemble!». Il ne s'en rend pas compte, mais il est déjà pleinement engagé dans le processus d'appropriation subjective du monde.

Malheureusement, certains parents réagissent à l'angoisse de leur enfant en étant encore plus angoissés; il y en a même qui font comme s'ils ne la voyaient pas, ou comme si celle-ci n'avait pas d'importance. Ils y répondent à côté, brouillent les repères de l'enfant, tentent de lui en faire adopter d'autres. Dans tous ces cas, l'enfant qui est confronté à une telle situation apprend à cacher ses émotions à ses proches, et pour mieux y parvenir, il se les cache aussi souvent à lui-même. Il se convainc qu'il « est nul », autrement dit qu'il est incapable de construire des représentations personnelles valides du monde; ou, pire encore, il imagine que son interlocuteur a des intentions sadiques à son égard.

On comprend que, lorsque de tels patients viennent en thérapie, ils soient particulièrement attentifs aux marques de compréhension de leur thérapeute. D'ailleurs, au début de leur traitement, ils accordent moins d'importance au fait de comprendre que de se savoir compris. Il leur faut d'abord prendre confiance en la valeur de leurs expériences émotionnelles et de leurs productions psychiques pour accéder, ensuite, au plaisir de les partager. Et, pour y parvenir, il est essentiel qu'ils rencontrent un thérapeute qui leur montre ses propres émotions. C'est ce qui leur permet de faire des expériences différentes de celles qu'ils ont vécues dans leur petite enfance, et ces expériences vont 
être à l'origine d'une façon différente d'envisager le monde et de s'envisager eux-mêmes. Leurs préoccupations authentiques et légitimes n'avaient pas été prises en compte dans leur petite enfance et ils en avaient déduit qu'elles n'étaient pas si légitimes que cela. Ils avaient pris l'habitude d'avoir chaud ou froid sans oser s'en plaindre, de ne jamais être certains qu'on les écoute, de renoncer à savoir s'ils avaient été bien compris, et finalement de faire ce qu'on leur demandait sans chercher à comprendre. En reconnaissant toutes ces préoccupations comme légitimes, le thérapeute leur rend le contentement qu'on éprouve normalement à être soi-même, qui avait été tari précocement chez eux.

\section{L'empathie pour mieux gérer les traumatismes}

Une attitude empathique du thérapeute ne permet pas seulement de remettre en route un processus de subjectivation entravé par des souffrances précoces. Elle joue aussi ce rôle lorsqu'un traumatisme grave l'a brutalement bloqué. Tout traumatisme grave s'accompagne en effet d'une triple rupture: dans l'estime que le sujet se porte à lui-même, dans la certitude de l'estime de ses proches, et dans le sentiment de faire partie d'une communauté qui l'accepte et l'intègre (Tisseron, 1992). La première de ces trois ruptures affecte ce que les psychanalystes appellent les investissements narcissiques, la seconde ce qu'ils appellent les investissements objectaux, et la troisième, ce que Bowlby a défini comme les liens d'attachement. Cette triple rupture implique si profondément les diverses couches de la personnalité qu'on ne guérit jamais totalement d'un traumatisme. Mais on le circonscrit, on le réduit, et on apprend à vivre avec ce qu'il en reste, ce qui n'est déjà pas si mal...

Commençons par la façon dont un traumatisme affecte l'estime de soi. C'est d'abord la déception de ne pas avoir su l'anticiper, et souvent aussi celle de ne pas avoir accompli les gestes qui auraient pu nous protéger ou protéger nos proches; c'est aussi l'angoisse de se découvrir plus vulnérable qu'on ne l'aurait cru, éventuellement d'avoir été vu dans une posture dégradante.

Cette brutale prise de conscience de ne pas avoir correspondu à son idéal s'accompagne fréquemment de l'inquiétude de perdre l'affection dont on était assuré jusque-là. Se sentir dévalorisé à ses propres yeux produit en effet l'inquiétude de l'être aussi aux yeux de ceux qu'on aime: «Mes proches m'accueilleront-ils encore comme l'un des leurs, quand ils sauront que je n'ai pas su me protéger ou, pire encore, protéger les miens? ». Sans compter les blessures physiques parfois liées au traumatisme: "Mon mari - ou ma femme - m'aimera-t-il toujours autant avec cette blessure?». 
Mais le traumatisme peut frapper encore une troisième dimension fondatrice du rapport à soi et au monde: lorsqu'il s'ajoute à l'angoisse de perdre l'affection de ceux qu'on aime, celle de perdre toute manifestation d'intérêt de qui que ce soit. Le traumatisme grave, celui qui confronte aux limites de l'humain, s'accompagne de l'angoisse d'être exclu du groupe dont on fait partie. Il peut s'agir du groupe familial, mais aussi de toutes les familles de substitution - syndicales, politiques, nationales —, et même, dans les cas extrêmes, de l'humanité entière. Il existe en effet des traumatismes dans lesquels on craint de perdre, même à ses propres yeux, la qualité d'être humain. La honte alors éprouvée est terriblement déstructurante et menaçante pour la socialisation. Les manières d'en parler sont d'ailleurs éclairantes: «J'ai envie de disparaître. J'ai envie de rentrer dans un trou de fourmi. Je préférerais ne pas exister. J'aurais voulu ne jamais être là ", etc. Lorsque ces trois dimensions sont affectées chez quelqu'un, il est en grand danger de se laisser mourir ou de se suicider. Mais ces situations sont heureusement rares, même si elles bordent tout vécu de honte.

Revenons maintenant à l'empathie. Ce sont justement les trois domaines menacés par le traumatisme qui sont pris en compte aussitôt que l'empathie est réciproque: la reconnaissance de l'autre et de soi comme susceptibles de s'aimer eux-mêmes pareillement; la reconnaissance de la possibilité d'aimer et d'être aimé exactement de la même manière; et la reconnaissance d'être tous deux sujets des mêmes droits et des mêmes devoirs dans la communauté des hommes. C'est la raison pour laquelle l'empathie réciproque permet de réaffilier à la communauté des hommes celui qui a craint d'en être marginalisé à l'occasion d'un traumatisme grave. D'autres moyens existent pour aider ces personnes: les livres, les émissions de radio faites par des psys, les sites d'échange d'expérience sur Internet, les thérapies familiales et de groupe, etc. Mais pour la plupart, elles ont besoin d'une psychothérapie individuelle.

Le thérapeute qui fait preuve d'une attitude empathique permet que se mette en place une situation dans laquelle son patient commence à se donner des représentations de ses traumatismes passés sans crainte de s'y retrouver seul. Dans le travail de coconstruction qui s'ensuit, ce qui relève de l'initiative de l'un et de l'autre est indécidable, tout comme est indécidable, dans le résultat, ce qui résulte de la part prise par chacun.

\section{De la psychanalyse envisagée comme un compagnonnage}

En pédagogie, une expression fait son chemin, celle de «compagnonnage », remise d'ailleurs à la mode par la pratique des jeux vidéo en réseau et 
la relation qui s'y instaure entre joueurs chevronnés et novices. Une relation de compagnonnage est mutuelle et réciproque, mais évidemment non symétrique. Pourquoi ne pas penser la thérapie analytique sur le même modèle? Chacun vient en effet à la relation avec ses attentes spécifiques et une idée de son interlocuteur. Autrement dit, chacun vient en étant porteur d'une image de l'interlocuteur virtuel qu'il s'attend à rencontrer. Du coup, l'analyste et le patient sont exactement dans la même situation, même s'ils n'ont pas les mêmes outils psychiques pour la gérer : ils doivent chacun se rendre disponible à une réalité forcément différente de l'idée qu'ils avaient des choses au départ. En pratique, cela signifie qu'ils doivent chacun faire évoluer l'image qu'ils ont de l'autre et de la relation au fur et à mesure de leur interrelation.

Bien entendu, un thérapeute empathique n'est pas forcément un bon thérapeute, mais un thérapeute froid et distant incite ses patients à s'éloigner de leurs propres émotions ou, au contraire, à les dramatiser pour y entraîner leur thérapeute. Dans les deux cas, ils s'éloignent d'eux-mêmes et aucune interprétation n'est capable de corriger cela. Au contraire, un thérapeute proche de ses propres émotions, et qui les montre, facilite la possibilité pour ses patients d'être proches d'eux-mêmes. Ils y acquièrent la possibilité de se remémorer les retraits émotionnels dont ils ont souffert dans leur petite enfance. Du coup, ils commencent à prendre de la distance par rapport au fait de rentrer dans leur coquille à la moindre déception. Leurs antennes d'escargot ne sont pas moins sensibles, mais ils sont devenus moins craintifs.

Le mot d'empathie désigne donc ce que nous essayons de faire, en tant que thérapeute, pour rendre à ces personnes le goût de l'échange vivant et leur permettre une existence plus facile. Ils s'y trouvent aidés dans les domaines qui ont été les moins explorés par Freud et qui sont devenus la cause principale de souffrance psychique: la construction d'une estime de soi adaptée permettant un attachement confiant au monde, et l'élaboration des traumatismes auxquels ils ont été confrontés. Pourtant, reconnaissons-le: il est impossible de compenser totalement auprès de tels patients ce qui leur a cruellement manqué dans leur petite enfance. La thérapie ne peut tout réparer, pas plus que la famille ou l'environnement. Au mieux, ils stabilisent et insèrent. Du point de vue d'une "guérison " possible, c'est peu, mais du point de vue de la vie, c'est déjà beaucoup!

Quant au thérapeute, souhaitons-lui de trouver dans ce cheminement partagé une issue nouvelle à ses conflits psychiques restés en suspens dans sa propre analyse. Leur travail commun les confortera alors tous deux dans le fait qu'il existe chez l'être humain d'autres forces que l'exaltation de soi et la 
recherche du pouvoir. C'est le désir de penser ensemble, de rêver ensemble, d'imaginer ensemble et de comprendre ensemble, qui sont autant de façons de se réconcilier à la fois avec les autres et avec soi-même, parce qu'on y reconnaît l'autre autant que l'on est reconnu par lui.

Bien sûr, le patient a souvent d'abord l'impression que cela ne correspond pas à l'image qu'il se faisait d'un psychanalyste, mais il s'en console vite en découvrant le plaisir qu'il prend à partager avec lui...

\author{
Serge Tisseron \\ serge.tisseron@gmail.com \\ www.sergetisseron.com
}

\title{
Notes
}

1. Film de Eric Toledano et Olivier Nakache, sorti en salle en novembre 2011.

2. Conférence faite à la société psychanalytique hongroise du cycle 1927-1928 portant sur "l'élasticité de la technique psychanalytique» où il parle de tact psychologique défini comme la faculté de «sentir avec», en utilisant le mot allemand Einfühlung). Ferenczi, S., Euvres complètes, IV, Paris: Payot, 1982.

3. Cette distinction fondée sur les catégories de la psychanalyse rejoint ce que le philosophe Axel Honneth appelle la reconnaissance. Elle fait intervenir pour lui aussi trois dimensions: la reconnaissance amoureuse, la reconnaissance juridique et la reconnaissance culturelle. La reconnaissance amoureuse fait intervenir l'amour au sens de rapports interpersonnels de proximité (liens familiaux, amicaux, amoureux). La reconnaissance juridique passe par le vecteur du contrat juridique conçu comme réciprocité entre les droits et les devoirs de chacun. Enfin, la reconnaissance culturelle est liée à la prestation ou la contribution qu'apportent les différents sujets qui composent la communauté à ses valeurs.

\section{Références}

Abraham, N., et Torok, M., 1978, Le touchant-touché introjectif, in L'écorce et le noyau, Paris, Aubier Flammarion.

Anzieu, D., 1979, La démarche de l'analyse transitionnelle en psychanalyse individuelle, in Psychanalyse des limites, Paris, Dunod, 2007.

Berthoz, A., et Jorland, G., 2004, L'empathie, Paris, Odile Jacob.

Bolognini, S., 2002, L'empathie psychanalytique, Paris, Eres, 2006.

De Waal, F., 2010, L'âge de l'empathie. Leçons de la nature pour une société solidaire, Paris, Les liens qui libèrent, 105.

Ehrenberg, A., 2004, Les changements de la relation normal-pathologique, À propos de la souffrance psychique et de la santé mentale, Esprit, n 304, 130-155.

Ferenczi, S., 1908-1912, Euvres complètes IV, Paris, Payot, 1982 (4 tomes).

Honneth, A., 2005, La réification. Petit traité de théorie critique, Paris, Gallimard, 2007.

Kohut, H., 1974, Le soi, Paris, PUF, 2004.

Rizzolatti, G., 2007, Les neurones miroirs, avec la collaboration Corrado Sinigaglia, traduit de l'italien par Marilène Raiola, Paris, Odile Jacob.

Tisseron, S., 1992, La honte, psychanalyse d'un lien social, Paris, Dunod. 
64 Filigrane, printemps 2014

Tisseron, S., 2010, L'empathie, au cour du jeu social, Paris, Albin Michel.

Tisseron, S., 2010, Le Jeu des Trois Figures en classes maternelles, Paris, Fabert, (téléchargeable sur http://www.yapaka.be).

Tisseron, S., 2013, Fragments d'une psychanalyse empathique, Paris, Albin Michel.

Trevarthen, C., Aitken, K.J., 2003, Intersubjectivité chez le nourrisson: recherche, théorie et application clinique, Devenir, $\mathrm{n}^{\circ}$ 4, 15, 309-428. 